\section{Effect of Metastable Liquid-Liquid Demixing on the Morphology of Nucleated Polymer Crystals}

\section{Wenbing Hu* and Daan Frenkel}

FOM Institute for Atomic and Molecular Physics, Kruislaan 407, 1098 SJ Amsterdam, The Netherlands

Received March 2, 2004

Revised Manuscript Received April 11, 2004

The first step in the transformation of a metastable parent phase into a more stable phase is the formation, by spontaneous fluctuations, of a nucleus that exceeds a critical threshold size. ${ }^{1}$ According to classical nucleation theory, the rate at which such nuclei form depends exponentially on the free energy cost needed to form a critical nucleus of the new phase. This free energy cost can be influenced by the vicinity of another phase transition through the presence of pretransitional fluctuations that occur near a critical point. In fact, simulations and theory suggest that the rate of crystal nucleation can be enhanced by the presence of density fluctuations associated with the presence of a (metastable) critical point. ${ }^{2-4}$ One would expect that any modification of the kinetics of crystal nucleation may significantly change the morphology of the resulting crystals. This should be true in particular in polymeric systems where the morphology of the resulting crystallites is very often determined by kinetics. An example is the formation of (metastable) lamellar crystallites: the chain folding observed in such crystals is completely dominated by the crystallization kinetics.

Indeed, experiments on polymer crystallization provide evidence that the morphology of the polymer crystals that form is dominated by the interplay of polymer crystallization and liquid-liquid demixing. ${ }^{5,6}$ It has even been suggested that this interplay between demixing and crystallization may be essential for the control of the morphology of polymer membranes. ${ }^{7}$

In this paper, we use dynamic Monte Carlo simulations to investigate effect of the vicinity of a liquidliquid demixing curve on the initiation of crystallization in polymer solutions and on the morphology of the resulting polymer crystallites.

To study this phenomenon, we make use of a simple lattice model that can account for both polymer crystallization and liquid-liquid demixing. ${ }^{8,9}$ In this model, we can independently tune the propensity of a polymer solution to crystallize and to undergo liquid-liquid demixing. In particular, the strength of the isotropic ("site-mixing") nearest-neighbor interaction determines the tendency of the system to undergo liquid-liquid demixing. On the other hand, the specific attraction between parallel nearest-neighbor bonds favors crystallization. ${ }^{8}$ In ref 9 we have shown that, through a judicious choice of these two interaction parameters, the liquid-liquid demixing transition can be made to occur either above or below the freezing temperature.

For the present study, we chose the conditions as follows: we fixed the polymer volume fraction at 0.150 , which is located in the vicinity of the critical concentration of 32-mer chains for liquid-liquid demixing. Then, we chose three sets of local interaction parameters such that in all cases the system had the same equilibrium

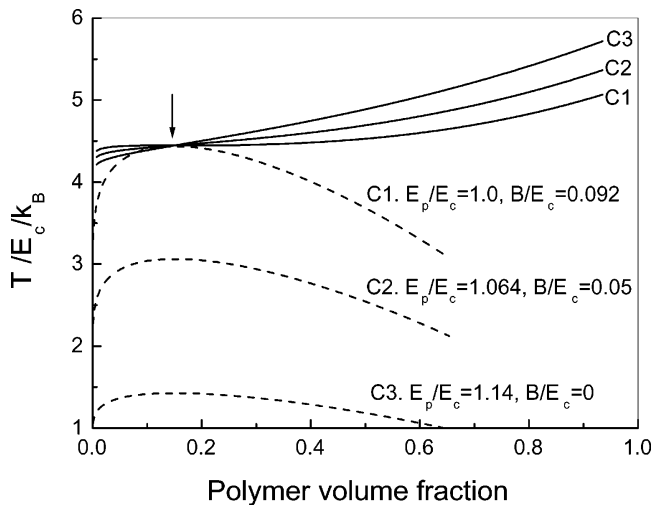

Figure 1. Liquid-solid coexistence curves (solid lines) and liquid-liquid coexistence curves (dashed lines) for the chains, each with 32 units, in a $64^{3}$ cubic lattice and with variable sets of interaction parameters as denoted. The curves are calculated from the mean-field theory (for the details, see ref 9). About the local interaction parameters, $E_{c}$ is the potential energy difference between collinear and noncollinear connections of two consecutive bonds along the chain, $B$ is the net potential energy exchange for a site-site contact between chain units and solvent, and $E_{p}$ is the potential energy deviation of those packings from a parallel packing of two neighboring bonds. $T$ is the temperature and $k_{B}$ is the Boltzmann constant. The arrow indicates the subsequent performance of simulations.

melting temperature but different critical temperatures for liquid-liquid demixing. We chose the highest of these demixing temperatures to be close to the freezing temperature (case C1 in Figure 1). The other two were located below the freezing curve with different depths (C2 and C3 in Figure 1). The choice of the interaction parameters was made on the basis of the mean-field theory of ref 9. The validity of the mean-field predictions has been tested by performing simulations with a crystallization template to suppress hysteresis at the freezing transition. ${ }^{9}$

In the present study we are specifically interested in nucleation. Hence, all simulations were performed on the solutions with the same concentrations and the same equilibrium melting temperatures. We cool ed all three samples bel ow the equilibrium melting point and monitored the onset of both liquid-liquid demixing and polymer crystallization. Since the initiation of polymer crystallization requires a certain supercooling, we can see how the metastable critical liquid-liquid demixing at different depths of supercooling changes the course of crystal nucleation.

Figure 2 shows the results of cooling processes. In the first case (C1) where the system has the critical point very close to the melting point, the liquid-liquid phase separation occurs first. This follows from Figure 2 as, upon cooling, the order parameter that monitors demixing changes well before the crystallinity order parameter starts to change. Subsequently, the system freezes. In fact, the onset of crystallization happens in this system at a higher temperature than in the other two systems. This indicates that the primary crystal nucleation is assisted by the prior liquid-liquid phase separation. In the second case (C2), the system has a somewhat lower liquid-liquid critical point than that in case $\mathrm{C} 1$. The corresponding cooling curves show that the crystal nucleation close to the liquid-liquid demix- 


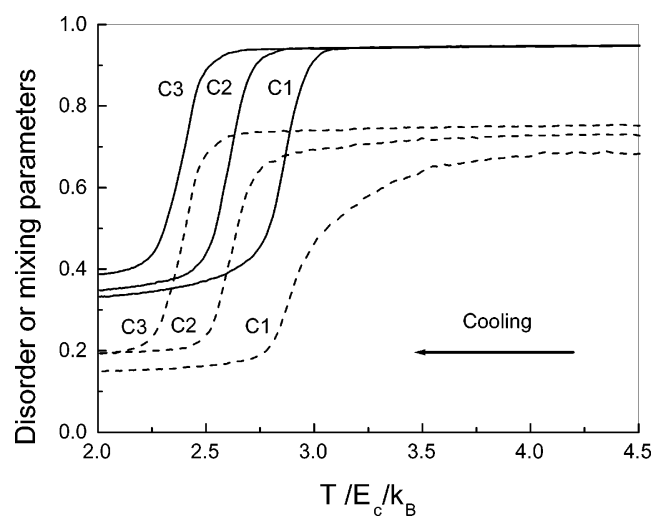

Figure 2. Cool ing curves of disorder parameters (solid lines) and mixing parameters (dashed lines) for the sample systems containing 1232 chains (polymer volume fraction 0.150 ) with the corresponding parameter sets in Figure 1. The disorder parameter is defined by the mean fraction of noncollinear connections along the chains, while the mixing parameter is defined by the mean fraction of neighboring sites around each chain unit occupied by the solvent. The curves are obtained from the simulations with a dynamic microrelaxation model ${ }^{3}$ and Metropolis sampling, where the potential energy barrier is $\Delta \mathrm{E}=\left(\mathrm{bB} / \mathrm{E}_{\mathrm{c}}-\mathrm{pE} \mathrm{E}_{\mathrm{p}} \mathrm{E}_{\mathrm{c}}-\mathrm{c}\right) \mathrm{E}_{\mathrm{c}}$ with the net changes in the number of mixing pairs between chain units and solvent (b), in the number of parallel-packing pairs of the crystallizable bonds ( $p)$, and in the number of collinear connections of consecutive bonds along the chains (c) in each step of microrelaxation. The systems with random coils are cooled by increasing the value of $E_{d}\left(k_{B} T\right)$ with a step length of 0.002 and a step period of $500 \mathrm{MC}$ cycles. One M onte Carlo (MC) cycle is defined as one trial move of each chain unit on an average. At each temperature step, the first $400 \mathrm{MC}$ cycles are discarded for fast equilibrating, and the remaining $100 \mathrm{MC}$ cycles are averaged for the reported data.

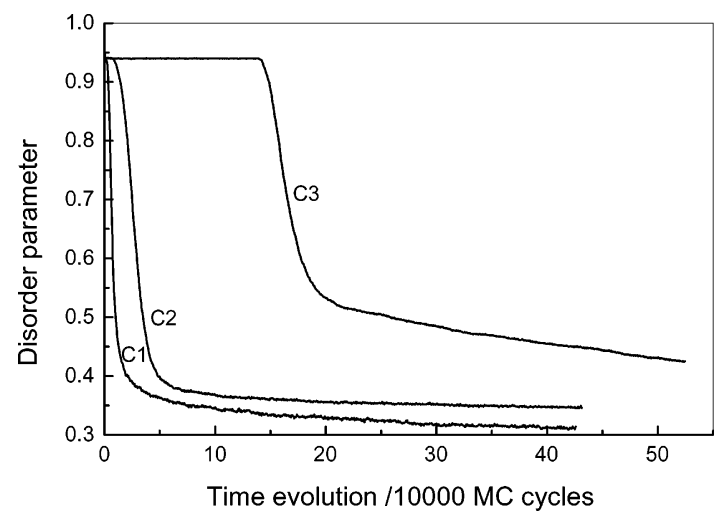

Figure 3. Time evolution curves of disorder parameters for the sample systems in Figure 2 quenched from the infinite temperature to $2.857 \mathrm{E} / \mathrm{k}_{\mathrm{B}}$. The lines are drawn on the data points with $500 \mathrm{MC}$ cycle interval.

ing temperature. In the third case (C3), the system has a very low critical point. The cooling curves show that the onset of crystallization is much higher than the metastable critical point. In fact, the onset of crystallization in this case happens at a temperature that is lower than in case C2. This suggests that in that case the vicinity of the liquid-liquid critical point does speed up the crystal nucleation process. No such influence is possible in case $C 3$. The overall trend shown in Figure 2 is consistent with the behavior found in simulations and theories of colloids and spherical proteins. ${ }^{2-4}$

Interestingly, we are able to see that the interplay between demixing and crystallization has a pronounced effect on the resultant crystallite morphology. To compare different crystallization pathways, we prepared all
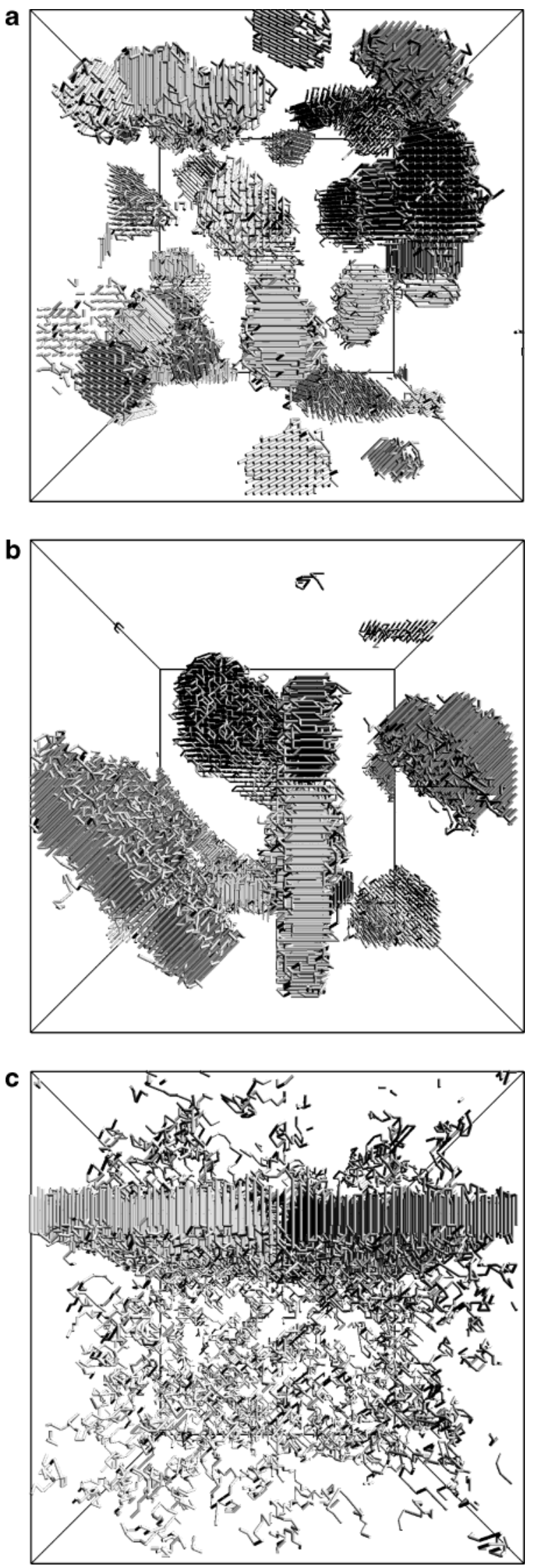

Figure 4. Snapshots of final crystallites after isothermal crystallization in Figure 3. The linear size of cubic box is 64 with periodic boundary conditions. All the bonds of 32-mer chains are drawn in cylinders. (a) Case $\mathrm{C} 1$, (b) case $\mathrm{C2}$, and (c) case C3. 
three model systems at high temperatures (where the polymers form random coils) and quenched the temperature to $2.857 \mathrm{E} d \mathrm{k}_{\mathrm{B}}$. At this temperature, we follow the isothermal crystallization process. Figure 3 shows the time evolution of disorder parameter. We can see that model C1 undergoes the fastest crystal nucleation, assisted by the prior liquid-liquid demixing. In the second case (C2), crystallization is slightly slower because here demixing does not precede crystallization but occurs simultaneously with it. The third case shows the sl owest crystallization. In fact, crystallization takes place suddenly, after a relatively long waiting time. This is typical for homogeneous crystal nucleation at high temperatures.

The resulting crystal morphologies show significant differences, as can be seen from Figure 4. Case C1 generates many small crystallites. This is to be expected, as the prior liquid-liquid spinodal demixing creates a spatially well-distributed inhomogeneous system. Rapid crystallization is confined to the regions of high density. In the second case, fewer and larger crystallites are found, as is to be expected in a system where density inhomogeneities have not yet fully developed before crystallization sets in. In the third case, only one crystallite forms. This indicates that, in this system, only one nucleation event took place. The resulting crystal nucleus then grew to become a single, lamellar crystal. Not all polymer is incorporated in this crystal, but the density of the remaining polymer coils is too low to cause subsequent crystal nucleation events.

In a summary, our simulations show that the primary nucleation of polymer crystals form in solutions can be significantly enhanced by prior liquid-liquid demixing and, to a lesser extent, by critical density fluctuations. This kinetic interplay controls the final crystallite morphology. Many small crystallites form if crystallization is induced by the prior liquid-liquid phase separation. The results of the present simulations would seem to be relevant for polymer crystallization experiments and for the control of crystallite morphology in many industrial processes.

Acknowledgment. The work of FOM institute is part of the research program of the "Stichting voor Fundamenteel Onderzoek der Materie" (FOM), which is financially supported by the "one Nederlandse organisatie voor Wetenschappelijk Onderzoek" (NWO).

\section{References and Notes}

(1) Kelton, K. F. In Crystal Nucleation in Liquids and Glasses; Ehrenreich, H., Turnbull, D., Eds.; Academic Press: Boston, 1991; Vol. 45.

(2) ten Wolde, P. R.; Frenkel, D. Science 1997, 277, 1975.

(3) Talanquer, V.; Oxtoby, D. W. J . Chem. Phys. 1998, 109, 223.

(4) Sear, R. P. J . Chem. Phys. 2001, 114, 3170.

(5) Inaba, N.; Sato, K.; Suzuki, S.; Hashimoto, T. Macromolecules 1986, 19, 1690.

(6) Schaaf, P.; Lotz, B.; Wittmann, J . C. Polymer 1987, 28, 193.

(7) Lee, H. K.; Myerson, A. S.; Levon, K. Macromolecules 1992, 25, 4002 and references therein.

(8) Hu, W.-B. J . Chem. Phys. 2000, 113, 3901.

(9) Hu, W.-B.; Frenkel, D.; Mathot, V. B. F. J . Chem. Phys. 2003, 118, 10343.

MA049581T 